\title{
ON SOME DENSITY TOPOLOGY WITH RESPECT TO AN EXTENSION OF LEBESGUE MEASURE
}

\author{
Katarzyna Flak — JACEK HeJduK — SylWia TOMCZYK
}

\begin{abstract}
This paper presents a density type topology with respect to an extension of Lebesgue measure involving sequence of intervals tending to zero. Some properties of such topologies are investigated.
\end{abstract}

Let $\mathbb{R}$ denote a set of real numbers, $\mathbb{N}$ a set of natural numbers, and $\lambda$ a Lebesgue measure on $\mathbb{R}$. By $\mathcal{L}$ we understand a family of Lebesgue measurable sets, by $\mathbb{L}$ a family of Lebesgue measurable null sets, and by $|I|$ a length of an interval $I$. By $\mu$ we denote any complete extension of Lebesgue measure $\lambda$, by $S_{\mu}$ a domain of function $\mu$, and by $\mathcal{I}_{\mu}$ a family of $\mu$-null sets. If $\mathcal{A}, \mathcal{B}$ are families of subsets of the space $\mathrm{X}$, then we use notation $\mathcal{A} \ominus \mathcal{B}=\{C \subset X$ : $C=A \backslash B, A \in \mathcal{A}, B \in \mathcal{B}\}$ and $\mathcal{A} \Delta \mathcal{B}=\{C \subset X: C=A \Delta B, A \in \mathcal{A}, B \in \mathcal{B}\}$, where $\Delta$ is an operation of the symmetric difference. It is well-known that if $\mathcal{A}$ is $\sigma$-algebra of sets in $X$ and $\mathcal{B}$ is $\sigma$-ideal of sets in $X$, then the family $\mathcal{A} \Delta \mathcal{B}$ is the smallest $\sigma$-algebra containing $\mathcal{A} \cup \mathcal{B}$.

It is clear that $x_{0} \in \mathbb{R}$ is a density point of a set $A \in \mathcal{L}$ if

It is equivalent to

$$
\lim _{h \rightarrow 0^{+}} \frac{\lambda\left(A \cap\left[x_{0}-h, x_{0}+h\right]\right)}{2 h}=1 .
$$

$$
\lim _{\substack{h_{1} \rightarrow 0^{+}, h_{2} \rightarrow 0^{+} \\ h_{1}+h_{2}>0}} \frac{\lambda\left(A \cap\left[x_{0}-h_{1}, x_{0}+h_{2}\right]\right)}{h_{1}+h_{2}}=1 .
$$

The above condition can be written as in [8]:

$$
\forall_{\left\{J_{n}\right\}_{n \in \mathbb{N}}}\left(0 \in \bigcap_{n \in \mathbb{N}} J_{n} \wedge\left|J_{n}\right| \underset{n \rightarrow \infty}{\longrightarrow} 0\right) \Longrightarrow \lim _{n \rightarrow \infty} \frac{\lambda\left(A \cap\left(J_{n}+x_{0}\right)\right)}{\left|J_{n}\right|}=1,
$$

where $\left\{J_{n}\right\}_{n \in \mathbb{N}}$ is a sequence of closed intervals.

(C) 2017 Mathematical Institute, Slovak Academy of Sciences.

2010 Mathematics Subject Classification: 54A10, 26A15, 54A20.

Keywords: $\mathcal{J}$-density topologies, $\mathcal{J}$-approximately continuous functions. 
Let $A \in \mathcal{L}$ and $\Phi_{d}(A)=\{x \in \mathbb{R}: x$ is a density point of $A\}$. Then the family $\mathcal{T}_{d}=\left\{A \in \mathcal{L}: A \subset \Phi_{d}(A)\right\}$ is a topology called density topology (see [8]). We say that a sequence of closed intervals $J=\left\{J_{n}\right\}_{n \in \mathbb{N}}$ is convergent to 0 if $\operatorname{diam}\left\{\{0\} \cup J_{n}\right\} \underset{n \rightarrow \infty}{\longrightarrow} 0$. We will consider sequences of closed intervals.

Let $J=\left\{J_{n}\right\}_{n \in \mathbb{N}}$ be a sequence of closed intervals convergent to zero. We say that the point $x_{0} \in \mathbb{R}$ is a $J$-density point of a set $A \in \mathcal{L}$, if

$$
\lim _{n \rightarrow \infty} \frac{\lambda\left(A \cap\left(J_{n}+x_{0}\right)\right)}{\left|J_{n}\right|}=1 .
$$

Let $\Phi_{J}(A)=\{x \in \mathbb{R}: x$ is a $J$-density point of a set $A\}$.

Having regard to the results of the paper [4, we obtain the following properties:

Property 1. If $A \in \mathcal{L}$, then $\Phi_{J}(A) \in \mathcal{F}_{\sigma \delta}$, so $\Phi_{J}(A) \in \mathcal{L}$.

Property 2. For arbitrary sets $A, B \in \mathcal{L}$ and arbitrary sequence $J$ of intervals convergent to zero, we obtain that

a) $\Phi_{J}(\mathbb{R})=\mathbb{R}, \Phi_{J}(\emptyset)=\emptyset$,

b) $\lambda(A \Delta B)=0 \Rightarrow \Phi_{J}(A)=\Phi_{J}(B)$,

c) $\Phi_{J}(A \cap B)=\Phi_{J}(A) \cap \Phi_{J}(B)$,

d) $\lambda\left(\Phi_{J}(A) \backslash A\right)=0$.

From the above property we conclude that for an arbitrary sequence of intervals tending to zero, operator $\Phi_{J}$ is an almost lower density operator on $(\mathbb{R}, \mathcal{L}, \mathbb{L})$ (see [3]).

The paper [4 also contains the proofs of the following two theorems:

TheOrem 1 (cf. [4). If $J=\left\{J_{n}\right\}_{n \in \mathbb{N}}$ is a sequence of intervals tending to zero, then the family

$$
\mathcal{T}_{J}=\left\{A \in \mathcal{L}: A \subset \Phi_{J}(A)\right\}
$$

is a topology such that $\mathcal{T}_{\text {nat }} \subsetneq \mathcal{T}_{J}$, where $\mathcal{T}_{\text {nat }}$ denotes a natural topology on $\mathbb{R}$. Topology $\mathcal{T}_{J}$ described above will be called the topology generated by the operator $\Phi_{J}$ on the space $(\mathbb{R}, \mathcal{L}, \mathbb{L})$.

For an arbitrary sequence $J=\left\{J_{n}\right\}_{n \in \mathbb{N}}$ tending to zero we define

$$
\alpha(J)=\limsup _{n \rightarrow \infty} \frac{\operatorname{diam}\left\{\{0\} \cup J_{n}\right\}}{\left|J_{n}\right|} .
$$

TheOrem 2 (cf. [4). If $J=\left\{J_{n}\right\}_{n \in \mathbb{N}}$ is a sequence of intervals tending to zero such that $\alpha(J)<\infty$, then for an arbitrary $A \in \mathcal{L}$,

$$
\lambda\left(\Phi_{J}(A) \Delta A\right)=0 .
$$




\section{ON SOME DENSITY TOPOLOGY...}

Taking into account Property 2 and Theorem 2, we conclude that if $\alpha(J)<\infty$, then operator $\Phi_{J}$ is the lower density operator on space $(\mathbb{R}, \mathcal{L}, \mathbb{L}$ ) (see [4]).

Let $J=\left\{J_{n}\right\}_{n \in \mathbb{N}}$ be a sequence of intervals tending to zero. Let $A \in S_{\mu}$. We say that a point $x_{0} \in \mathbb{R}$ is a $J$-density point of the set $A$ if

$$
\lim _{n \rightarrow \infty} \frac{\mu\left(A \cap\left(J_{n}+x_{0}\right)\right)}{\left|J_{n}\right|}=1 .
$$

Let $\Phi_{J}^{\mu}(A)=\{x \in \mathbb{R}: x$ is a $J$-density point of the set $A\}$.

Property 3 (cf. [4]). For an arbitrary sequence of intervals $J=\left\{J_{n}\right\}_{n \in \mathbb{N}}$ tending to zero and for an arbitrary set $A \in S_{\mu}$ we obtain that $\Phi_{J}^{\mu}(A) \in \mathcal{L}$.

P r o o f. Let $A \in S_{\mu}$. Then, $x \in \Phi_{J}^{\mu}(A)$ if and only if

$$
\forall_{k \in \mathbb{N}} \exists_{m \in \mathbb{N}} \forall_{n>m} \frac{\mu\left(A \cap\left(J_{n}+x\right)\right)}{\left|J_{n}\right|} \geq 1-\frac{1}{k} .
$$

Hence,

$$
\Phi_{J}^{\mu}(A)=\bigcap_{k \in \mathbb{N}} \bigcup_{m \in \mathbb{N}} \bigcap_{n>m}\left\{x \in \mathbb{R}: \frac{\mu\left(A \cap\left(J_{n}+x\right)\right)}{\left|J_{n}\right|} \geq 1-\frac{1}{k}\right\} .
$$

A function $f(x)=\mu\left(A \cap\left(J_{n}+x\right)\right)$ is continuous for a fixed $n \in \mathbb{N}$, furthermore, it satisfies Lipschitz's condition. In fact, for every $x_{1}, x_{2}$ we have

$$
\begin{aligned}
\left|f\left(x_{1}\right)-f\left(x_{2}\right)\right| & =\left|\mu\left(A \cap\left(J_{n}+x_{1}\right)\right)-\mu\left(A \cap\left(J_{n}+x_{2}\right)\right)\right| \\
& \left.\leq \mid \mu\left(A \cap\left(\left(J_{n}+x_{1}\right)\right) \Delta\left(J_{n}+x_{2}\right)\right)\right) \mid \\
& \left.\leq \mid \mu\left(\left(J_{n}+x_{1}\right)\right) \Delta\left(J_{n}+x_{2}\right)\right) \mid \\
& \leq 2\left|x_{1}-x_{2}\right| .
\end{aligned}
$$

Hence, $\Phi_{J}^{\mu}(A) \in F_{\delta \sigma}$, so in particular, $\Phi_{J}^{\mu}(A) \in \mathcal{L}$.

Directly from the definition of the operator $\Phi_{J}^{\mu}$, we can deduce the following property.

Property 4. For any sequence of intervals $J=\left\{J_{n}\right\}_{n \in \mathbb{N}}$ tending to zero and any sets $A, B \in S_{\mu}$ we obtain:

a) $\Phi_{J}^{\mu}(\mathbb{R})=\mathbb{R}, \Phi_{J}^{\mu}(\emptyset)=\emptyset$,

b) $\Phi_{J}^{\mu}(A \cap B)=\Phi_{J}^{\mu}(A) \cap \Phi_{J}^{\mu}(B)$,

c) $\mu(A \Delta B)=0 \Rightarrow \Phi_{J}^{\mu}(A)=\Phi_{J}^{\mu}(B)$.

Now, we prove another property of the operator $\Phi_{J}^{\mu}$ for a sequence of intervals $J=\left\{J_{n}\right\}_{n \in \mathbb{N}}$ tending to zero and such that $0 \in J_{n}$ for any $n \in \mathbb{N}$. Let $\mathfrak{I}^{0}$ denote a family of all sequences of intervals tending to zero and containing zero. 


\section{KATARZYNA FLAK — JACEK HEJDUK — SYLWIA TOMCZYK}

Theorem 3. If $J \in \mathfrak{I}^{0}$, then for any $A \in S_{\mu}$ such that $A \subset \Phi_{J}^{\mu}(A)$ we obtain

$$
\mu\left(\Phi_{J}^{\mu}(A) \backslash A\right)=0 .
$$

P r o o f. Let $A \subset \mathbb{R}$ be bounded. So, there exists an interval $K$ such that $A \subset K$. Now, we show that for any $0<\varepsilon<\lambda(K)$ the family

$$
\mathcal{K}(\varepsilon)=\left\{H \subset K: \mu(H \cap A)>\left(1-\frac{\varepsilon}{\lambda(K)}\right) \lambda(H)\right\},
$$

where $H$ is a closed interval, is a Vitali cover of the set $A$.

This is a consequence of the fact that if $x \in A$, then $x \in \Phi_{J}^{\mu}(A)$, namely,

$$
\forall_{\eta>0} \exists_{\delta>0} \forall_{\left|J_{n}\right|<\delta} \frac{\mu\left(A \cap\left(J_{n}+x\right)\right)}{\lambda\left(J_{n}\right)}>1-\eta .
$$

Putting $\eta=\frac{\varepsilon}{\lambda(\mathcal{K})}$, we find arbitrary short intervals such that $\left(J_{n}+x\right) \in \mathcal{K}(\varepsilon)$, and $x$ belongs to the interval $\left(J_{n}+x\right)$ for any $n \in \mathbb{N}$.

Hence, from the Vitali theorem there exists a sequence of closed, pairwise disjoint intervals $\left\{P_{n}\right\}_{n \in \mathbb{N}}$ from the family $\mathcal{K}(\varepsilon)$ such that

Then,

$$
\lambda\left(A \backslash \bigcup_{n=1}^{\infty} P_{n}\right)=0
$$

$$
\mu\left(\bigcup_{n=1}^{\infty}\left(P_{n} \backslash A\right)\right) \leq \sum_{n=1}^{\infty} \mu\left(P_{n} \backslash\left(A \cap P_{n}\right)\right) \leq \frac{\varepsilon}{\lambda(\mathcal{K})} \sum_{n=1}^{\infty} \lambda\left(P_{n}\right) \leq \varepsilon \frac{\lambda(\mathcal{K})}{\lambda(\mathcal{K})}=\varepsilon
$$

Let

$$
C=\left(A \backslash \bigcup_{n=1}^{\infty} P_{n}\right) \cup \bigcup_{n=1}^{\infty} P_{n}
$$

Then, $A \subset C, C$ is Lebesgue measurable set and $\mu(C \backslash A)<\varepsilon$. Therefore,

so,

$$
\forall_{n \in \mathbb{N}} \exists_{\substack{C_{n} \in \mathcal{L} \\ A \subset C_{n}}} \mu\left(C_{n} \backslash A\right)<\frac{1}{n},
$$

$$
A \subset \bigcap_{n=1}^{\infty} C_{n} \text { and } \mu\left(\bigcap_{n \in \mathbb{N}} C_{n} \backslash A\right)=0
$$

Then,

$$
A=\bigcap_{n \in \mathbb{N}} C_{n} \backslash\left(\bigcap_{n \in \mathbb{N}} C_{n} \backslash A\right) .
$$

Putting

$$
B=\bigcap_{n=1}^{\infty} C_{n}, \quad D=\bigcap_{n=1}^{\infty} C_{n} \backslash A,
$$

we obtain

$$
A=B \backslash D, \quad \text { where } \quad B \in \mathcal{L} \quad \text { and } \quad \mu(D)=0 \text {. }
$$


At the same time, $\mu\left(\Phi_{J}^{\mu}(A) \backslash A\right)=0$, because $\mu\left(\Phi_{J}^{\mu}(A) \backslash A\right)=\mu\left(\Phi_{J}^{\mu}(B \backslash D) \backslash\right.$ $(B \backslash D))=\mu\left(\Phi_{J}^{\mu}(B) \backslash B\right)=\lambda\left(\Phi_{J}(B) \backslash B\right)$. If $J \in \mathfrak{I}^{0}$, then $\alpha(J)=1$, so from Theorem 2, we obtain $\lambda\left(\Phi_{J}(B) \backslash B\right)=0$.

Theorem 4. If $J \in \mathfrak{I}^{0}$, then the family

$$
\mathcal{T}_{J}^{\mu}=\left\{A \in S_{\mu}: A \subset \Phi_{J}^{\mu}(A)\right\}
$$

is a topology on $\mathbb{R}$ containing topology $\mathcal{T}_{d}$.

P r o of. By Property 4 , we obtain that $\emptyset, \mathbb{R} \in \mathcal{T}_{J}^{\mu}$, and $\mathcal{T}_{J}^{\mu}$ is closed under intersection. Let $\left\{A_{t}\right\}_{t \in T} \subset \mathcal{T}_{J}^{\mu}$. We show that $\bigcup_{t \in T} A_{t} \in \mathcal{T}_{J}^{\mu}$. The pair $\left(S_{\mu}, \mathcal{I}_{\mu}\right)$ satisfies ccc, because the measure $\mu$ is $\sigma$-finite, so it has the hull property. Let $B \subset \bigcup_{t \in T} A_{t}$ be a measurable kernel. Then, $\mu\left(\left(B \cap A_{t}\right) \Delta A_{t}\right)=0$. Hence,

$$
B \subset \bigcup_{t \in T} A_{t} \subset \bigcup_{t \in T} \Phi_{J}^{\mu}\left(A_{t}\right)=\bigcup_{t \in T} \Phi_{J}^{\mu}\left(B \cap A_{t}\right) \in \Phi_{J}^{\mu}(B)
$$

but $\mu\left(\Phi_{J}^{\mu}(B) \backslash B\right)=0$, then $\bigcup_{t \in T} A_{t} \in S_{\mu}$, and consequently, $\bigcup_{t \in T} A_{t} \subset$ $\Phi_{J}^{\mu}\left(\bigcup_{t \in T} A_{t}\right)$, which means that $\bigcup_{t \in T} A_{t} \in \mathcal{T}_{J}^{\mu}$. Obviously, $\mathcal{T}_{d} \subset \mathcal{T}_{J}^{\mu}$.

TheOrem 5. For any sequence $J \in \mathfrak{I}^{0}$ we have

$$
\mathcal{T}_{J}^{\mu}=\mathcal{T}_{J} \ominus \mathcal{I}_{\mu},
$$

where $\mathcal{T}_{J}$ is a topology generated by the operator $\Phi_{J}$ on the space $(\mathbb{R}, \mathcal{L}, \mathbb{L})$.

P r o o f. Let $A=B \backslash C, B \in \mathcal{T}_{J}, C \in \mathcal{I}_{\mu}$. Then, $A \in S_{\mu}$ and $\Phi_{J}^{\mu}(A)=\Phi_{J}^{\mu}(B \backslash C)=$ $\Phi_{J}^{\mu}(B) \supset B \supset B \backslash C=A$, so $A \in \mathcal{T}_{J}^{\mu}$.

Let $A \in \mathcal{T}_{J}^{\mu}$, then $A \in S_{\mu}$ and $A \subset \Phi_{J}^{\mu}(A)$. From the proof of Theorem 3 , we have

$$
A=D \backslash E, \quad \text { where } \quad D \in \mathcal{L} \text { and } E \in \mathcal{I}_{\mu} \text {. }
$$

At the same time, $A=\Phi_{J}^{\mu}(A) \backslash\left(\Phi_{J}^{\mu}(A) \backslash A\right)=\Phi_{J}^{\mu}(D \backslash E) \backslash F=\Phi_{J}^{\mu}(D) \backslash F=$ $\Phi_{J}(D) \backslash F$, where $F=\Phi_{J}^{\mu}(A) \backslash A$. Since $J \in \mathfrak{I}^{0}$, then $\alpha(J)=1$, so $\Phi_{J}$ is the lower density operator on $(\mathbb{R}, \mathcal{L}, \mathbb{L})$. It implies that $\Phi_{J}\left(\Phi_{J}(D)\right)=\Phi_{J}(D)$. Hence, $\Phi_{J}(D) \in \mathcal{T}_{J}$. From Theorem 3 we have $F \in \mathcal{I}_{\mu}$, so $A \in \mathcal{T}_{J} \ominus \mathcal{I}_{\mu}$.

Now, we quote a theorem on extension of measure.

Theorem 6 (see [6]). Let $(X, S, \mu)$ be a measurable space with a $\sigma$-finite measure $\mu$. Let $J \subset 2^{X}$ be a $\sigma$-ideal such that $\mu_{*}(B)=0$ for $B \in J$, where $\mu_{*}$ is an inner measure induced by $\mu$. Then, the function $\mu^{\prime}$ defined on $\sigma$-field $S \Delta J$ by $\mu^{\prime}(A \Delta B)=\mu(A)$, where $A \in S, B \in J$, is an extension of the measure $\mu$, and if $\mu$ is a complete measure, then $\mu^{\prime}$ is a complete measure as well.

Corollary 1. If $\mu$ is a complete extension of Lebesgue measure, then the function $\mu^{\prime}$ defined on $\sigma$-field $\mathcal{L} \Delta \mathcal{I}_{\mu}$, where $\mathcal{I}_{\mu}$ is $\sigma$ ideal of $\mu$-zero sets by $\mu^{\prime}(A \Delta B)=$ $\lambda(A)$, where $A \in \mathcal{L}, B \in \mathcal{I}_{\mu}$, is a complete extension of Lebesgue measure. 
Let $\mu$ be a complete extension of Lebesgue measure. Let us recall the definition of the density operator on the space $\left(\mathbb{R}, S_{\mu}, \mathcal{I}_{\mu}\right)$.

Definition 1. We shall say that operator $\Phi: S_{\mu} \rightarrow 2^{X}$ is the lower density operator on the space $\left(\mathbb{R}, S_{\mu}, \mathcal{I}_{\mu}\right)$ if the following conditions are satisfied:

a) $\Phi(\mathbb{R})=\mathbb{R}, \Phi(\emptyset)=\emptyset$,

b) $\forall_{A, B \in S_{\mu}} \Phi(A \cap B)=\Phi(A) \cap \Phi(B)$,

c) $\forall_{A, B \in S_{\mu}} \mu(A \Delta B)=0 \Rightarrow \Phi(A)=\Phi(B)$,

d) $\forall_{A \in S_{\mu}} \mu(A \Delta \Phi(A))=0$.

Similarly to Theorem 4, we can prove the following theorem (see 4]).

ThEOREM 7. If $\Phi: S_{\mu} \rightarrow 2^{\mathbb{R}}$ is the lower density operator on $\left(\mathbb{R}, S_{\mu}, \mathcal{I}_{\mu}\right)$, then the family $\mathcal{T}_{\Phi}=\left\{A \in S_{\mu}: A \subset \Phi(A)\right\}$ is a topology on $\mathbb{R}$.

We now justify that topology $\mathcal{T}_{J}^{\mu}$ generated by operator $\Phi_{J}^{\mu}$ can also be generated by some lower density operator on the space $\left(\mathbb{R}, \mathcal{L} \Delta \mathcal{I}_{\mu}, \mathcal{I}_{\mu}\right)$.

TheOREM 8. If $J \in \mathfrak{I}^{0}$ and $\mathcal{T}_{J}^{\mu}$ is a topology generated by the operator $\Phi_{J}^{\mu}$, then there exists a complete extension $\mu^{\prime}$ of Lebesgue measure such that $S_{\mu^{\prime}} \subset S_{\mu}$, $\mathcal{I}_{\mu^{\prime}}=\mathcal{I}_{\mu}$, and $\Phi_{\mu^{\prime}}=\left.\Phi_{J}^{\mu}\right|_{S_{\mu^{\prime}}}$, where $\left.\Phi_{J}^{\mu}\right|_{S_{\mu^{\prime}}}$ denotes the restriction of $\Phi_{J}^{\mu}$ to the family $S_{\mu^{\prime}}$, is the lower density operator on $\left(\mathbb{R}, S_{\mu^{\prime}}, \mathcal{I}_{\mu^{\prime}}\right)$ and topology $\mathcal{T}_{\mu^{\prime}}=$ $\left\{A \in S_{\mu^{\prime}}: A \subset \Phi_{\mu^{\prime}}(A)\right\}$ is identical to the topology $\mathcal{T}_{J}^{\mu}$.

Proof. Let $\mu^{\prime}$ be a measure defined on $\sigma$-field $S_{\mu^{\prime}}=\mathcal{L} \Delta \mathcal{I}_{\mu}$ as in Corollary 1 , Then $S_{\mu^{\prime}} \subset S_{\mu}$, and clearly $\mathcal{I}_{\mu^{\prime}}=\mathcal{I}_{\mu}$. To prove that $\Phi_{\mu^{\prime}}=\left.\Phi_{J}^{\mu}\right|_{S_{\mu^{\prime}}}$ is the lower density operator on $\left(\mathbb{R}, S_{\mu^{\prime}}, \mathcal{I}_{\mu^{\prime}}\right)$ by Property 4 , it is sufficient to show condition d) from Definition 1 Let $A \in S_{\mu^{\prime}}$, then $A=B \Delta C$, where $B \in \mathcal{L}, C \in \mathcal{I}_{\mu}$. At the same time, $\Phi_{\mu^{\prime}}(A) \Delta A=\Phi_{\mu^{\prime}}(B \Delta C) \Delta(B \Delta C)=\Phi_{J}^{\mu}(B) \Delta B \Delta C$. By Theorem 2 we get $\Phi_{J}^{\mu}(B) \Delta B \in \mathcal{I}_{\mu}$, so $\Phi_{\mu}(A) \Delta A \in \mathcal{I}_{\mu}$. Hence, $\Phi_{\mu}(A) \Delta A \in \mathcal{I}_{\mu^{\prime}}$. We show that $\mathcal{T}_{J}^{\mu}=\mathcal{T}_{\mu^{\prime}}$. Let $A \in \mathcal{T}_{J}^{\mu}$. Then, by Theorem 5 we have $A=B \backslash C$, where $B \in \mathcal{L}$ and $C \in \mathcal{I}_{\mu}$. Since $\mathcal{I}_{\mu}=\mathcal{I}_{\mu^{\prime}}$, we get that $A \in S_{\mu^{\prime}}$ and $\Phi_{\mu^{\prime}}(A)=$ $\Phi_{\mu^{\prime}}(B \backslash C)=\Phi_{\mu^{\prime}}(B)=\Phi_{J}^{\mu}(B)=\Phi_{J}^{\mu}(B \backslash C)=\Phi_{J}^{\mu}(A) \supset A$. Hence, $A \in \mathcal{T}_{\mu^{\prime}}$. Let $A \in \mathcal{T}_{\mu^{\prime}}$. Then, $A \in S_{\mu^{\prime}}$ and $A \subset \Phi_{\mu^{\prime}}(A)$. Clearly, $A \in S_{\mu}$, because $S_{\mu^{\prime}} \subset S_{\mu}$ and $\Phi_{\mu^{\prime}}(A)=\Phi_{J}^{\mu}(A)$. Thus, $A \in \mathcal{T}_{J}^{\mu}$.

If $J \in \mathfrak{I}^{0}$, then, by the previous theorem, it follows that the topology $\mathcal{T}_{J}^{\mu}$ coincides with the topology generated by the operator $\left.\Phi_{J}^{\mu}\right|_{\mathcal{L} \Delta \mathcal{I}_{\mu}}$ which is the lower density operator on $\left(\mathbb{R}, \mathcal{L} \Delta \mathcal{I}_{\mu}, \mathcal{I}_{\mu}\right)$. Hence in the light of the properties of an abstract density topology generated by the lower density operator which are presented in Theorem 25.3 and Theorem 25.9 in [3], we obtain the following theorem. 
Theorem 9. Let $J \in \mathfrak{I}^{0}$. Let $\mathcal{T}_{J}^{\mu}$ be a topology generated by an operator $\Phi_{J}^{\mu}$ on $\left(\mathbb{R}, S_{\mu}, \mathcal{I}_{\mu}\right)$. Then:

a) $A \in \mathcal{I}_{\mu}$ if and only if $A$ is $\mathcal{T}_{J}^{\mu}$-closed and $\mathcal{T}_{J}^{\mu}$-nowhere dense;

b) If $A \in \mathcal{I}_{\mu}$, then $A$ is $\mathcal{T}_{J}^{\mu}$-closed and $\mathcal{T}_{J}^{\mu}$-discrete;

c) $\mathcal{I}_{\mu}=\mathcal{K}\left(\mathcal{T}_{J}^{\mu}\right)$, where $\mathcal{K}\left(\mathcal{T}_{J}^{\mu}\right)$ is a family of the first category sets with respect to topology $\mathcal{T}_{J}^{\mu}$

d) $\operatorname{Bor}\left(\mathcal{T}_{J}^{\mu}\right)=B\left(\mathcal{T}_{J}^{\mu}\right)=\mathcal{L} \Delta \mathcal{I}_{\mu}$, where $\operatorname{Bor}\left(\mathcal{T}_{J}^{\mu}\right)$ is a family of Borel sets, $B\left(\mathcal{T}_{J}^{\mu}\right)$ is a family of Baire sets with respect to the topology $\mathcal{T}_{J}^{\mu}$;

e) $\left(\mathbb{R}, \mathcal{T}_{J}^{\mu}\right)$ is a Baire space;

f) $A$ is $\mathcal{T}_{J}^{\mu}$-compact if and only if $A$ is finite;

g) $\left(\mathbb{R}, \mathcal{T}_{J}^{\mu}\right)$ is not a first countable, not a second countable, and not a separable space;

h) $\left(\mathbb{R}, \mathcal{T}_{J}^{\mu}\right)$ is not a Lindelöf space;

i) $\operatorname{int}_{\mathcal{T}_{\Phi_{J}^{\mu}}^{\mu}}(A)=A \cap \Phi_{J}^{\mu}(\mathcal{K} A), A \subset \mathbb{R}, \mathcal{K} A$ - $\mu$-measurable kernel of the set $\mathrm{A}$;

j) $\operatorname{int}_{\mathcal{T}_{\Phi_{J}^{\mu}}^{\mu}}(A)=A \cap \Phi_{J}^{\mu}(A), A \in S_{\mu}$.

As a consequence of the previous theorem, we obtain that in the case of a measure $\mu$ such that $S_{\mu}=\mathcal{L} \Delta \mathcal{I}_{\mu}$ and a sequence $J \in \mathfrak{I}^{0}$, topology $\mathcal{T}_{J}^{\mu}$ generated by the operator $\Phi_{J}^{\mu}$ is such that $B\left(\mathcal{T}_{J}^{\mu}\right)=\mathcal{L} \Delta \mathcal{I}_{\mu}, \mathcal{K}\left(\mathcal{T}_{J}^{\mu}\right)=\mathcal{I}_{\mu},\left(\mathbb{R}, \mathcal{T}_{J}^{\mu}\right)$ is a Baire space. It is easy to see that the family of nonempty $\mathcal{T}_{J}^{\mu}$-open and pairwise disjoint sets is at most countable. Hence, in the case $S_{\mu}=\mathcal{L} \Delta \mathcal{I}_{\mu}$, topology $\mathcal{T}_{J}^{\mu}$ is a von Neumann topology associated with the measure $\mu$ (see [8]). We can prove the following theorem.

TheOREM 10. Let $J \in \mathfrak{I}^{0}$ and let and $\mathcal{T}_{J}^{\mu}$ be a topology generated by the operator $\Phi_{J}^{\mu}$. Then, the next conditions are equivalent:

a) $\Phi_{J}^{\mu}$ is a lower density operator on $\left(\mathbb{R}, S_{\mu}, \mathcal{I}_{\mu}\right)$;

b) $S_{\mu}=\mathcal{L} \Delta \mathcal{I}_{\mu}$;

c) $\mathcal{T}_{J}^{\mu}$ is a von Neumann topology with respect to the measure $\mu$.

Proof. $a) \Rightarrow b$ ) Of course, $\mathcal{L} \Delta \mathcal{I}_{\mu} \subset S_{\mu}$. Let $A \in S_{\mu}$. Then, $A \Delta \Phi_{J}^{\mu}(A) \in \mathcal{I}_{\mu}$. From Property 3 we have $\Phi_{J}^{\mu}(A) \in \mathcal{L}$, so $A \in \mathcal{L} \Delta \mathcal{I}_{\mu}$.

The implication $b) \Rightarrow c$ ) is a consequence of the previous theorem.

We prove the implication $c) \Rightarrow a$ ). By Property 4 , it suffices to prove that $A \Delta \Phi_{J}^{\mu}(A) \in \mathcal{I}_{\mu}$ for $A \in S_{\mu}$.

If $\mathcal{T}_{J}^{\mu}$ is a von Neumann topology with respect to the measure $\mu$, then $S_{\mu}=$ $B\left(\mathcal{T}_{J}^{\mu}\right)=\mathcal{L} \Delta \mathcal{I}_{\mu}$ so $A \in \mathcal{L} \Delta \mathcal{I}_{\mu}$. Hence $A=B \Delta C$, where $B \in \mathcal{L}$ and $C \in \mathcal{I}_{\mu}$. Therefore $B \Delta C \Delta \Phi_{J}^{\mu}(B \Delta C)=B \Delta \Phi_{J}^{\mu}(B) \Delta C \in \mathcal{I}_{\mu}$, because $B \Delta \Phi_{J}^{\mu}(B) \in \mathbb{L}$ by Theorem 2 . 


\section{KATARZYNA FLAK — JACEK HEJDUK — SYLWIA TOMCZYK}

Now, we discuss some results related separation axioms.

For any sequence $J \in \mathfrak{I}^{0}$ we have that $\mathcal{T}_{\text {nat }} \subset \mathcal{T}_{J}^{\mu}$. Hence, the space $\left(\mathbb{R}, \mathcal{T}_{J}^{\mu}\right)$ is Hausdorff. Paper [2] demonstrates that the space $\left(\mathbb{R}, \mathcal{T}_{J}\right)$ is regular, and in paper [5] it was shown that $\left(\mathbb{R}, \mathcal{T}_{J}\right)$ is completely regular for any sequence of intervals $J$ tending to zero such that $\alpha(J)<\infty$. So, for any sequence $J \in \mathfrak{I}^{0}$, the space $\left(\mathbb{R}, \mathcal{T}_{J}\right)$ is regular.

Investigating paper [7, we get that for $J \in \mathfrak{I}^{0}$ the family of $\mathcal{T}_{J}$-continuous functions and $\mathcal{T}_{J}^{\mu}$-continuous functions with value in a topological regular space are equal. It implies that if $\left(\mathbb{R}, \mathcal{T}_{J}\right)$ is a regular space, then the space $\left(\mathbb{R}, \mathcal{T}_{J}^{\mu}\right)$ is regular if and only if $\mathcal{T}_{J}^{\mu}=\mathcal{T}_{J}$. Finally, we get

Property 5. For any sequence $J \in \mathfrak{I}^{0}$, the following conditions are equivalent:

a) $\left(\mathbb{R}, \mathcal{T}_{J}^{\mu}\right)$ is completely regular;

b) $\left(\mathbb{R}, \mathcal{T}_{J}^{\mu}\right)$ is regular;

c) $\mathcal{T}_{J}^{\mu}=\mathcal{T}_{J}$

d) $\mathcal{I}_{\mu}=\mathbb{L}$.

P r o o f. $a \Rightarrow b$ is obvious. Let $\left(\mathbb{R}, \mathcal{T}_{J}^{\mu}\right)$ be regular. Let $A \in \mathcal{T}_{J}^{\mu}$. Then $A=V \backslash B$, if $V \in \mathcal{T}_{J}$ and $B \in \mathcal{I}_{\mu}$. Let us assume that $A \notin \mathcal{T}_{J}$. Then, $B \in \mathcal{I}_{\mu} \backslash \mathcal{L}$. Let $C$ be a measurable hull of $B$. We see that $\Phi_{J}(C) \backslash B \neq \emptyset$ because otherwise $\Phi_{J}(C) \subset$ $B \subset C$ would be measurable. Let $x \in \Phi_{J}(C) \backslash B$. Since the space $\left(\mathbb{R}, \mathcal{T}_{\mu}\right)$ is regular and $B$ is $\mathcal{T}_{\mu}$-closed, then there exist $V_{1}, V_{2} \in \mathcal{T}_{\mu}, V_{1} \cap V_{2}=\emptyset$ and $x \in V_{1}$, $B \subset V_{2}$. Since $V_{1}=W_{1} \backslash D_{1}, V_{2}=W_{2} \backslash D_{2}$, where $W_{1}, W_{2} \in \mathcal{T}_{J}, D_{1}, D_{2} \in \mathcal{I}_{\mu}$ we get $W_{1} \cap W_{2}=\emptyset$. Then, $W_{1} \subset \mathbb{R} \backslash W_{2}$ and $C \cap W_{1} \subset C \backslash W_{2} \subset C \backslash B$. From the definition of a hull, $\lambda\left(C \backslash W_{2}\right)=0$. This implies that $\Phi_{J}\left(C \cap W_{1}\right)=$ $\Phi_{J}(C) \cap \Phi_{J}\left(W_{2}\right)=\emptyset$. At the same time, $x \in \Phi_{J}(C) \cap W_{1} \subset \Phi_{J}(C) \cap \Phi_{J}\left(W_{2}\right)=\emptyset$, which is a contradiction. Since $\mathcal{T}_{J} \subset \mathcal{T}_{J}^{\mu}$, implication $b \Rightarrow c$ has been proved. Let $\mathcal{T}_{J}^{\mu}=\mathcal{T}_{J}$ and $A \in \mathcal{I}_{\mu} \backslash \mathbb{L}$. Then, $X \backslash A \in \mathcal{T}_{J}^{\mu}$. Hence $X \backslash A \in \mathcal{T}_{J}$ and finally, $A \in \mathcal{L}$. So, $A \in \mathbb{L}$. Since $\mathbb{L} \subset \mathcal{I}_{\mu}$, implication $c \Rightarrow d$ has been proved. If $\mathcal{I}_{\mu}=\mathbb{L}$, then $\mathcal{T}_{J}^{\mu}=\mathcal{T}_{J}$. Since $J \in \mathcal{I}^{0}$, then by Theorem 13 in $[5],\left(\mathbb{R}, \mathcal{T}_{J}^{\mu}\right)$ is completely regular.

It is worth mentioning that there exist extensions of the Lebesgue measure maintaining Lebesgue null sets.

Theorem 11 (cf. [1). Under the Continuum Hypothesis there exists a nonseparable extension of the Lebesgue measure on $\mathbb{R}$ whose null sets coincide with the null sets of the Lebesgue measure.

TheOREm 12. Let $J \in \mathfrak{I}^{0}$. Then the space $\left(\mathbb{R}, \mathcal{T}_{J}^{\mu}\right)$ is not normal. 


\section{ON SOME DENSITY TOPOLOGY...}

P r o of. By Theorem 3.15 in [2], we conclude that the space $\left(\mathbb{R}, \mathcal{T}_{J}\right)$ is not normal. Hence there are $\mathcal{T}_{J}$-closed sets $F_{1}, F_{2} \neq \emptyset$ and $F_{1} \cap F_{2}=\emptyset$ such that for any open set $V_{1}, V_{2} \in \mathcal{T}_{J}$ such that $F_{1} \subset V_{1}, F_{2} \subset V_{2}$, we obtain $V_{1} \cap V_{2} \neq \emptyset$. The sets $F_{1}, F_{2}$ are also $\mathcal{T}_{J}^{\mu}$-closed. In case $\left(\mathbb{R}, \mathcal{T}_{J}^{\mu}\right)$ is a normal space, then there are sets $W_{1}, W_{2} \in \mathcal{T}_{J}^{\mu}$ such that $F_{1} \subset W_{1}, F_{2} \subset W_{2}, W_{1} \cap W_{2}=\emptyset$. Since by the form of the topology $\mathcal{T}_{J}^{\mu}, W_{1}=V_{1} \backslash Z_{1}, W_{2}=V_{2} \backslash Z_{2}$, where $V_{1}, V_{2} \in \mathcal{T}_{J}$ and $Z_{1}, Z_{2} \in \mathcal{I}_{\mu}$. We conclude that $V_{1}, V_{2}$ are disjoint and also $F_{1} \subset V_{1}, F_{2} \subset V_{2}$. This contradiction finishes the proof.

\section{REFERENCES}

[1] KHARAZISHVILI, A. B.: A nonseparable extension of the Lebesgue measure without new nullsets, Real Anal. Exchange 33 (2007/2008), 259-268.

[2] HEJDUK, J.-LORANTY, A.-WIERTELAK, R.: J-approximately continuous functions, Tatra Mt. Math. Publ. 62 (2015), 45-55.

[3] HEJDUK, J.-WIERTELAK, R.: On the abstract density topologies generated by lower and almost lower density operators, in: Traditional and present-day topics in real analysis (M. Filipczak et al., eds.), Łódź University Press, Łódź 2013, pp. 431-447.

[4] HEJDUK, J.-WIERTELAK, R.: On the generalization of the density topologies on the real line, Math. Slovaca 64 (2014), 1267-1276.

[5] HEJDUK, J.-WIERTELAK, R.: On some properties of J-approximately continuous functions, Math. Slovaca (to appear).

[6] MARCZEWSKI, E.: Sur l'extension de la measure lebesguienne, Fund. Math. 25 (1935), $551-558$.

[7] MARTIN, N. F. G.: Generalized condensation points, Duke Math. J. 28 (1961), 507-5014.

[8] OXTOBY, J. C.: Measure and Category, 2nd edition. Graduate Texts in Math. Vol. 2, Springer-Verlag, Berlin, 1980.

Received February 25, 2017

\author{
Eódź University \\ Faculty of Mathematics and \\ Computer Science \\ Banacha 22 \\ PL-90-238 Łódź \\ POLAND \\ E-mail: flakk@math.uni.lodz.pl \\ hejduk@math.uni.lodz.pl \\ sylwia.tomczyk.12z@gmail.com
}

